Dept. of Meat Hygien and Technology,

Fac. Vet. Med., Albaath University.

\title{
DETECTION OF LEAD ELEMENT IN CATTLE AND SHEEP MEAT IN HOMS GOVERNORATE
}

(With 4 Tables and 4 Figures)

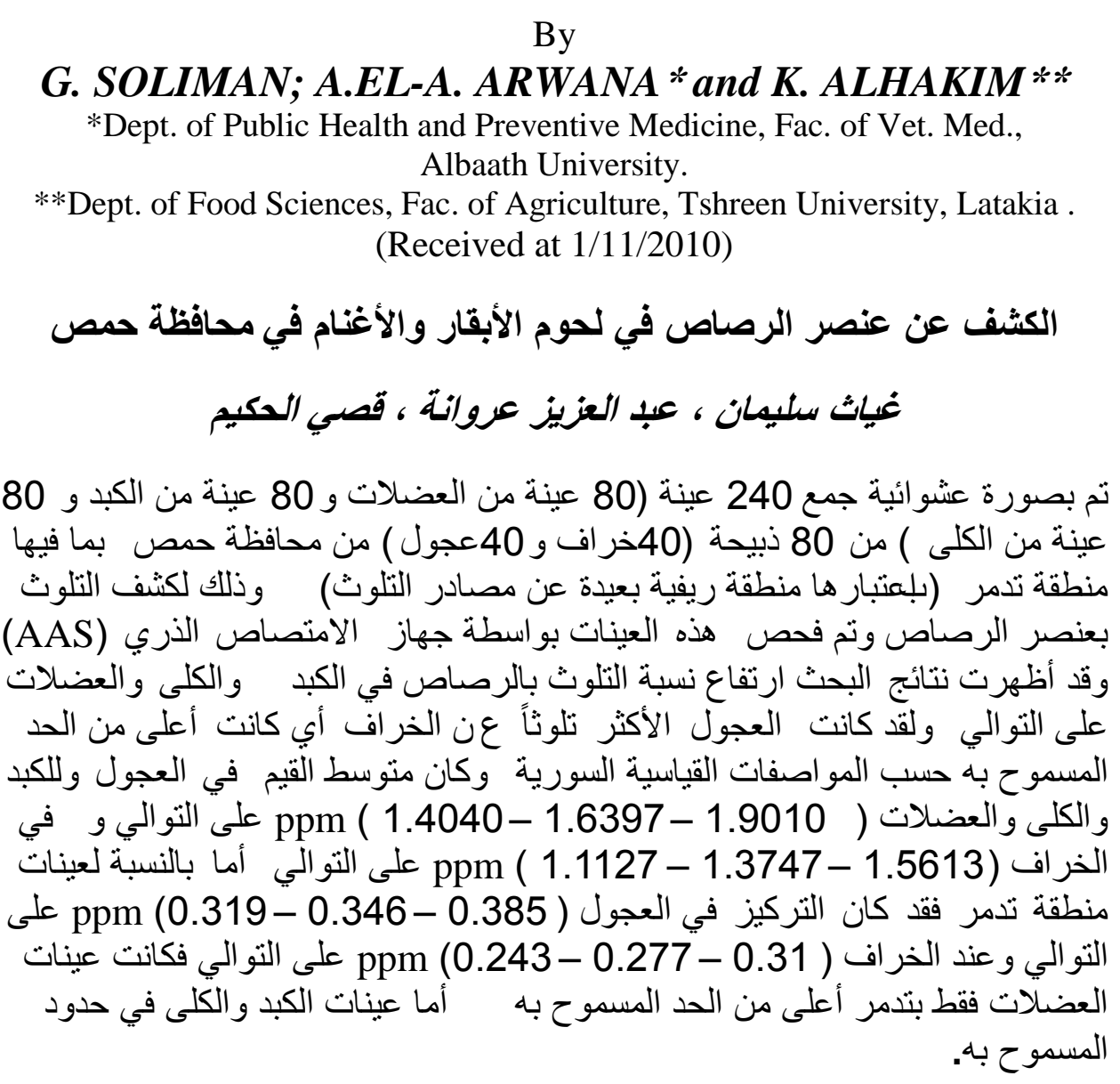

\section{SUMMARY}

The study was conducted on 240 random samples ( 80 for each of muscle, liver and kidney), which were collected from 80 carcasses (40 male sheep and 40 calves) from Homs Governorate including Palmyra region because it is considered rural region far away from contamination resources. For detection contamination by lead samples were tested using atomic absorbation technique and the results showed a proportional increasl of 
lead concentration in liver, kidney, and muscles respectively. Contaminated level was higher in calves comparing to male sheep, this means the proportion was greater than the critical limit according to Syrian standard measures. The average of values in liver ,kidney and muscle of calves were $1.4040,1.6397$ and $1.9010 \mathrm{mg} / \mathrm{kg}$ respectively and in male sheep were $1.5613,1.1127$ and $1.3747 \mathrm{mg} / \mathrm{kg}$ respectively. The mean concentration in Palmyra samples in liver, kidney and muscle of calves were $0.319-0.346$ and $0.385 \mathrm{mg} / \mathrm{kg}$ and in sheep male were $0.243-0.277$ and $0.31 \mathrm{mg} / \mathrm{kg}$. Muscle samples in Palmyra region only were greater than the permissible limit while liver and kidney were within the permissible limit

Key words:

\section{INTRODUCTION المقدم-ة}

يزداد خطر التلوث بالرصاص في العالم عام بعد عام بسبب ازدهار التكنولوجيا

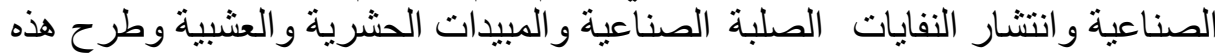

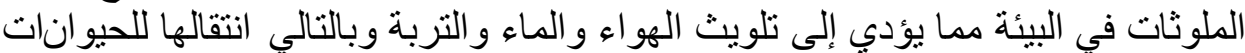

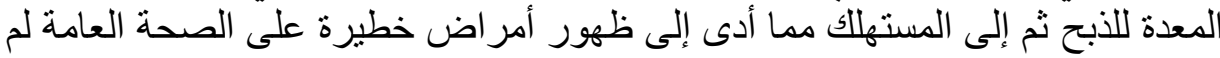

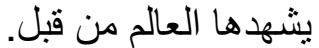
إن تلوث البيئة بهذه الملوثات و انتقالها إلى الحيوانات

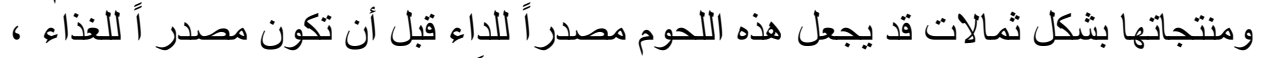

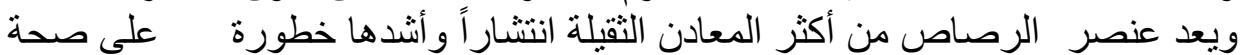

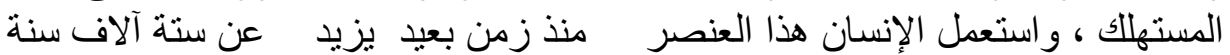

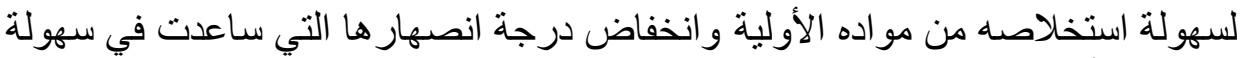
تشكيله بالأشكال المطلوبة. (Eisinger, 1996).

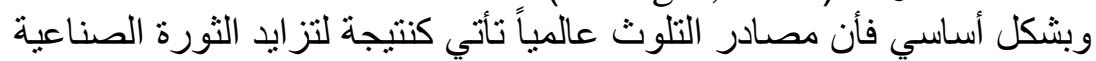

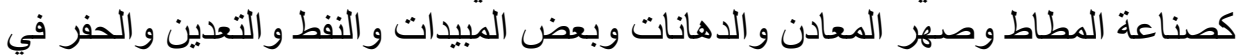

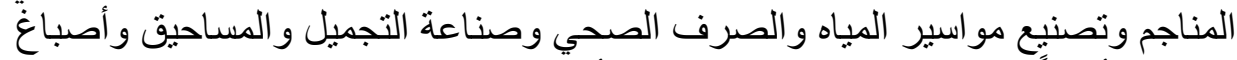

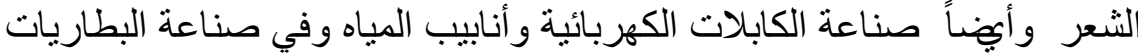

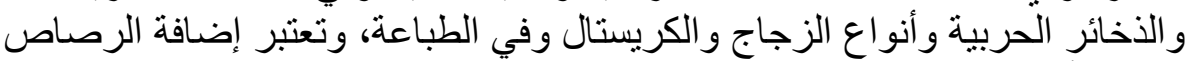

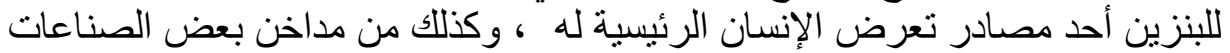

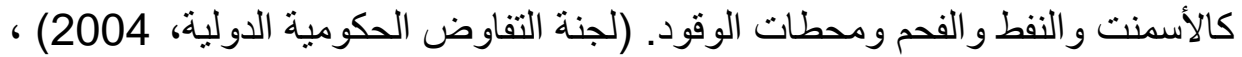
(WHO ، 2000) (الأسبوع الثقافي الكيميائي الخامس ، ، 2003). ويعزى وجود تركيز ات عالية

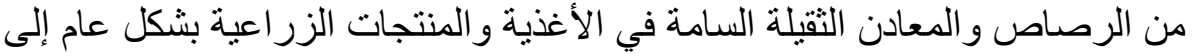
تلوث التربة في المنطقة الملوثة (فرونتاسيفا وآخرون ،2005). 


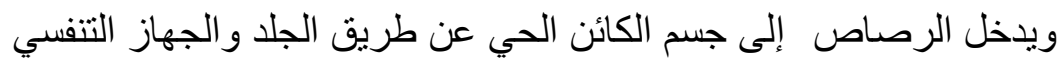

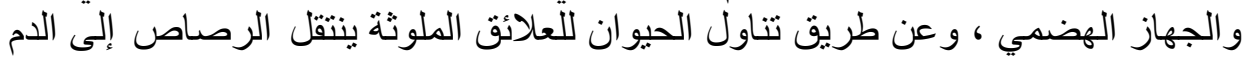

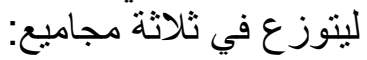

مجاميع التبادل السريع (الدم و القلب و الرئتين و الكبد و الكليتين و الدماغ و والقناة

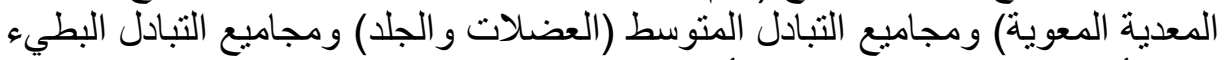
و التي أثبت وجودها في العظام و الأسنان. (Wright et al., 2003) .

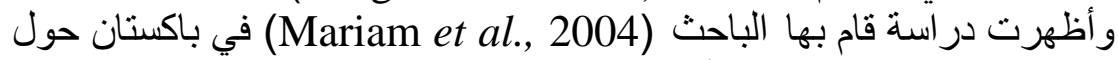

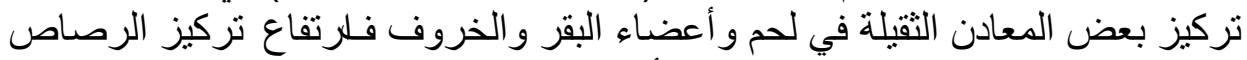

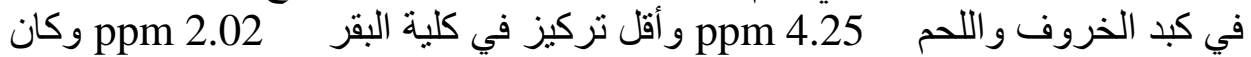

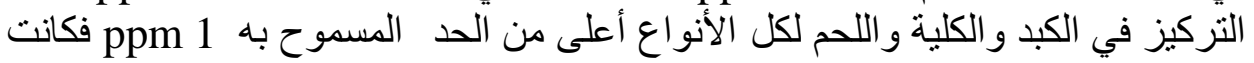

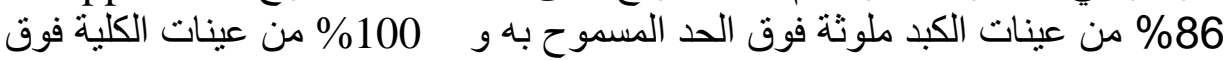

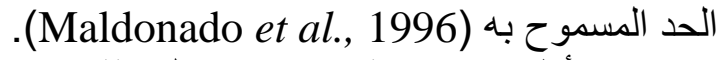

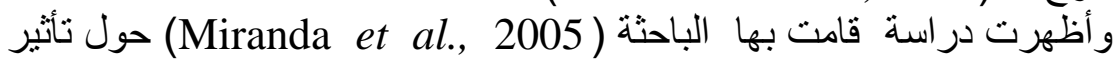
التلوث بالعناصر السامة على العجول في اسبانيا في منطقة صناعة إنية (شمال اسبانيا) وفي

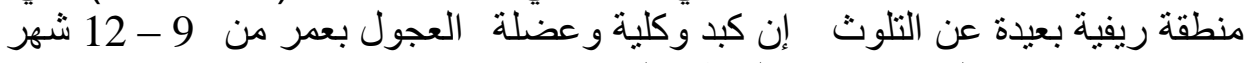

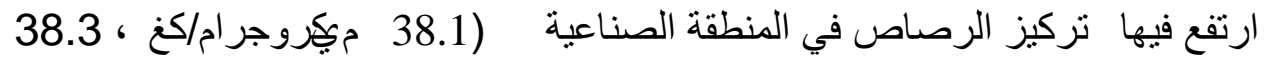

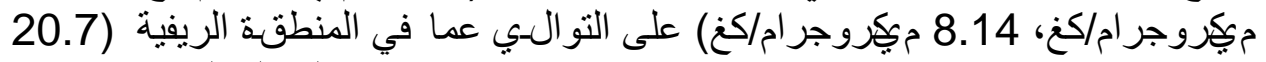

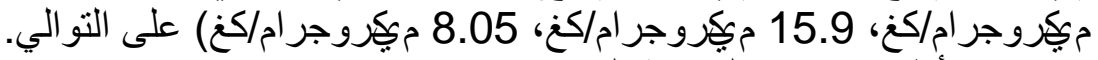

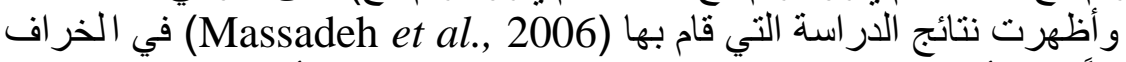

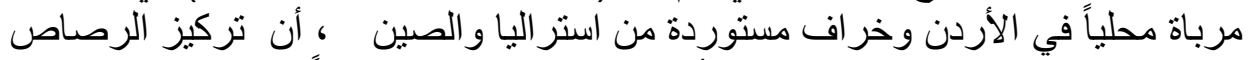

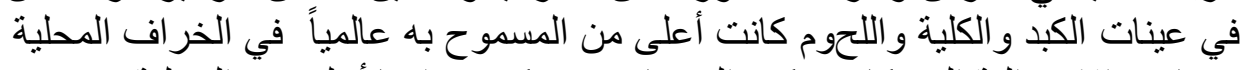

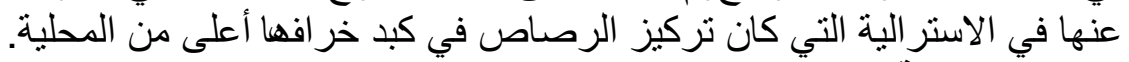

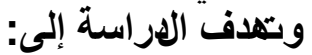

1 - تقدير نسب الرصاص الرف في لحوم وكبد وكلى الأغنام والأبقار بمحافظة حمص.

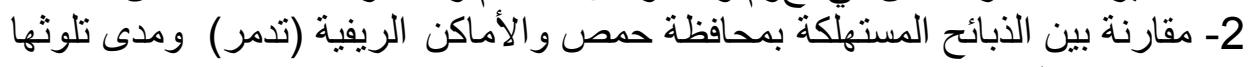

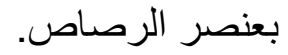

\section{MATERIALS and METHOD مواد وطراثق البحث}

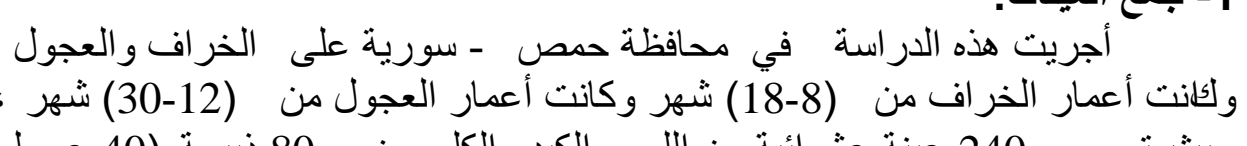

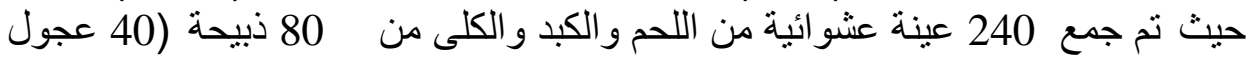

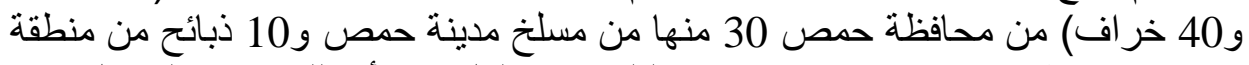

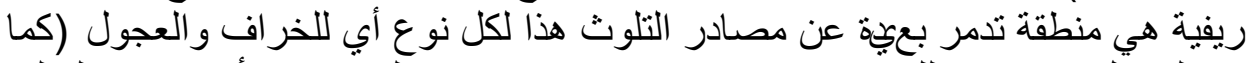

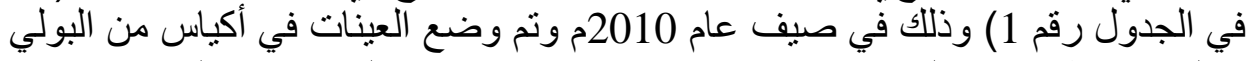

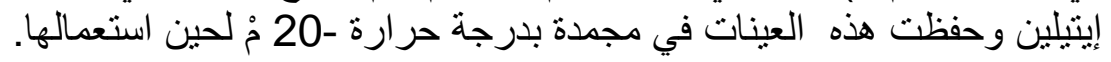


الجدول رقم 1: يوضح عدد ونوع ومنطقة العينات و الذبائح

\begin{tabular}{|c|c|c|c|c|c|c|}
\hline \multicolumn{2}{|c|}{ المنطقة } & \multirow{2}{*}{ الذبائح } & \multicolumn{3}{|c|}{ نوع العينات و عددها } & \multirow{2}{*}{ نوع الحيو ان والعمر } \\
\hline تدمر & حمص & & عضلات & كلية & كبد & \\
\hline 10 & 30 & 40 & 40 & 40 & 40 & خر اف(8- 18) شهر \\
\hline 10 & 30 & 40 & 40 & 40 & 40 & عجول(12-30) شهر \\
\hline \multicolumn{6}{|c|}{240 عينة } & المجموع \\
\hline
\end{tabular}

2- المواد الكيميائية والمواد الكاثفة للبحث:

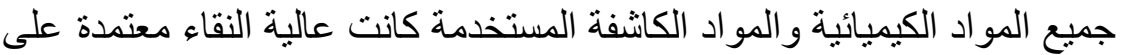

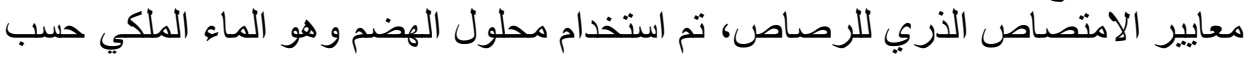

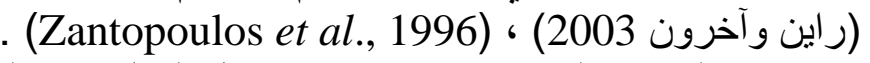

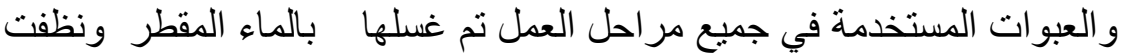

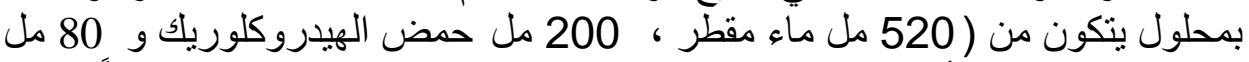

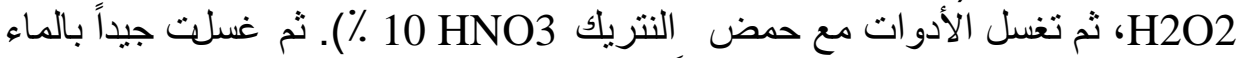
المقطر وجففت بالهو اء في حاضنة بعيداً عن أي مصدر من مصادر التلوث أو أو الغبار .(El-Mowafi,1995)

و العينات تم تحليلها وتقدير مستوى الرصاص فيها باستخدام جهاز التحليل

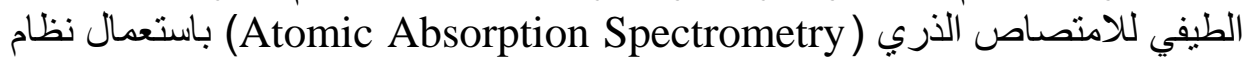
اللهب من نوع شيماتزو (AA6800) على لمبة رصاص نوع ( BGC-D2) وطول

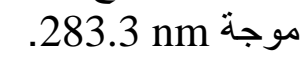
3 - التحليل المخبري: التهن اـ هضم العينات: نم هضم العينات لكل من الكبد و الكلى و العضلات وذللك طبقاً لطريقة (Seady, 2001 ; Tsoumbaris, 1990)

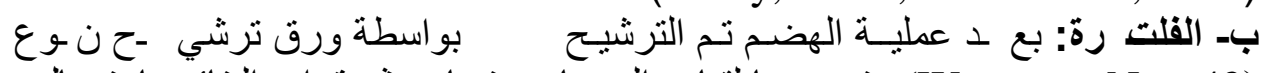
و وتجهيز ها لقياس الرصاص فيها، ثم قراءة النتائج باستعمال (Wattman No. 42) جهاز الامتصاص الذري (AAS) وذللك طبقاً لطريقة (A.O.A.C,1990).

\section{RESULTS}

النتائج 


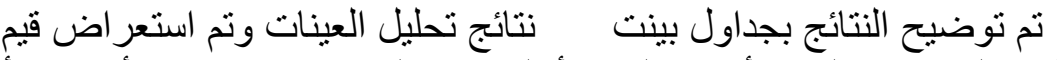
المتوسطات الحسابية والحد الأدنى و الحد الأعلى وحد الثقة

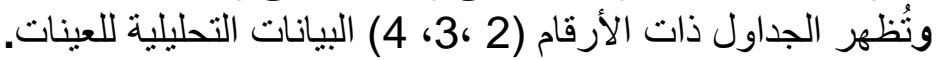

الجدول رقم 2: البيانات التحليلية لعينات العضلات و الكلى و الكبد في الخر اف بمحافظة حمص ومنطقة تدمر بppm و عدد العينات بين قوسين.

\begin{tabular}{|c|c|c|c|c|}
\hline حد الثقة 95\% الأصغري & و الحد الأعظمي الأدنى & الانحر اف المعياري الحسبي & و مصدر أخذ العينات & المدروسة \\
\hline $1.4509-0.7745$ & $3.27-0.05$ & $0.9057 \pm 1.1127$ & حمص(30) & \multirow[t]{2}{*}{ العضلة } \\
\hline $0.3363-0.1497$ & $0.52-0.04$ & $0.2430 * \pm 0.1304$ & تدمر(10) & \\
\hline $1.7604-0.9890$ & $4.11-0.29$ & $1.0329 \pm 1.3747$ & حمص(30) & \multirow[t]{2}{*}{ الكلية } \\
\hline $0.3768-0.1772$ & $0.55-0.12$ & $0.2770 * \pm 0.1394$ & تدمر (10) & \\
\hline $4081-0.34$ & $4081-0.34$ & $1.1514 \pm 1.5613$ & حمص(30) & \multirow[t]{2}{*}{ الكبد } \\
\hline $0.4174-0.2026$ & $0.61-0.11$ & $0.3100 * \pm 0.1502$ & تدمر (10) & \\
\hline \multicolumn{5}{|c|}{$<0.05^{*}$} \\
\hline
\end{tabular}

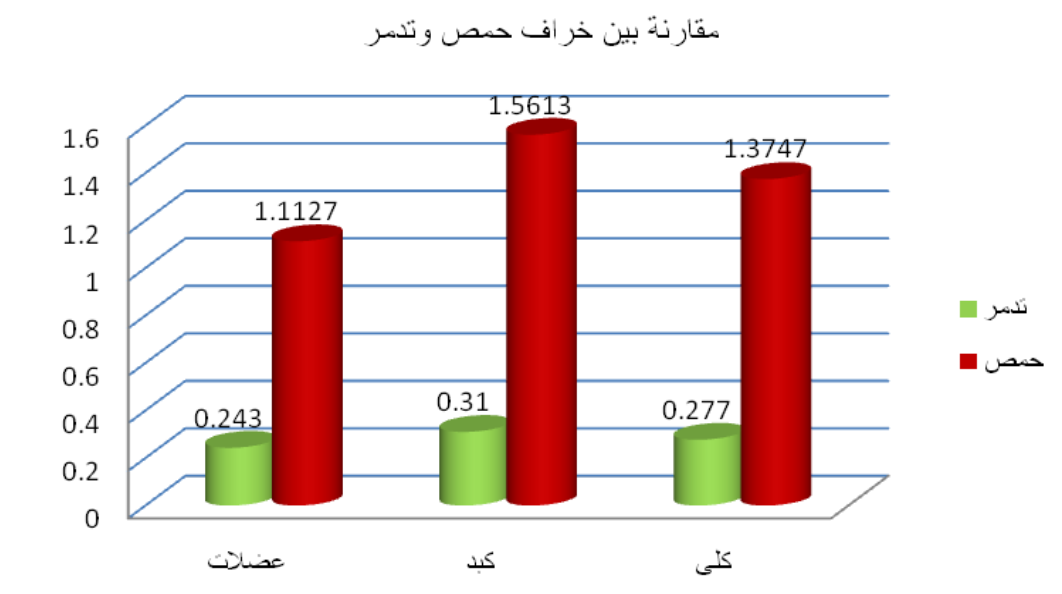

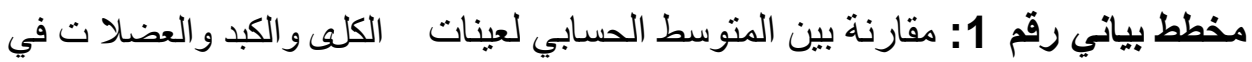

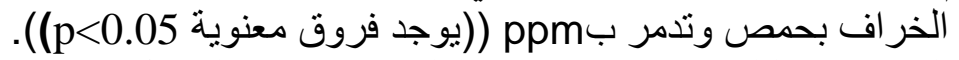

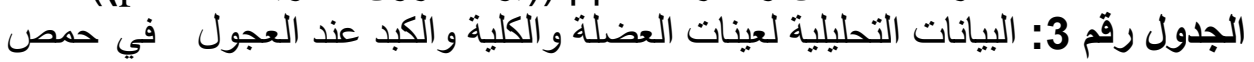
و وتدمر بppm و عدد العينات بين قوسين. 
Assiut Vet. Med. J. Vol. 57 No. 128 January 2011

\begin{tabular}{|c|c|c|c|c|}
\hline حد الثقة95\% الأصغري & الحد الأدنى و الحد & الانحتر اف المعيابيدي & مصدر أخذ & الأعضاء \\
\hline $1.8113-0.9967$ & $4.11-0.07$ & $1.0908 \pm 1.4040$ & حمص(30) & \multirow[t]{2}{*}{ العضلة } \\
\hline $0.4125-0.2255$ & $0.48-0.06$ & $0.1308 \pm * 0.3190$ & تدمر(10) & \\
\hline $2.1005-1.1788$ & $4.65-0.23$ & $1.2343 \pm 1.6397$ & حصص(30) & \multirow[t]{2}{*}{ الكلية } \\
\hline $0.4443-0.2477$ & $0.52-0.16$ & $0.1374 \pm 0.3460 *$ & تدمر(10) & \\
\hline $2.4152-1.3868$ & $5.07-0.29$ & $1.3770 \pm 1.9010$ & حمص(30) & \multirow[t]{2}{*}{ الكبد } \\
\hline $0.4874-0.2826$ & $0.55-0.18$ & $0.1431 \pm * 0.3850$ & تدمر(10) & \\
\hline \multicolumn{5}{|c|}{$\mathrm{p}<0.05^{*}$} \\
\hline
\end{tabular}

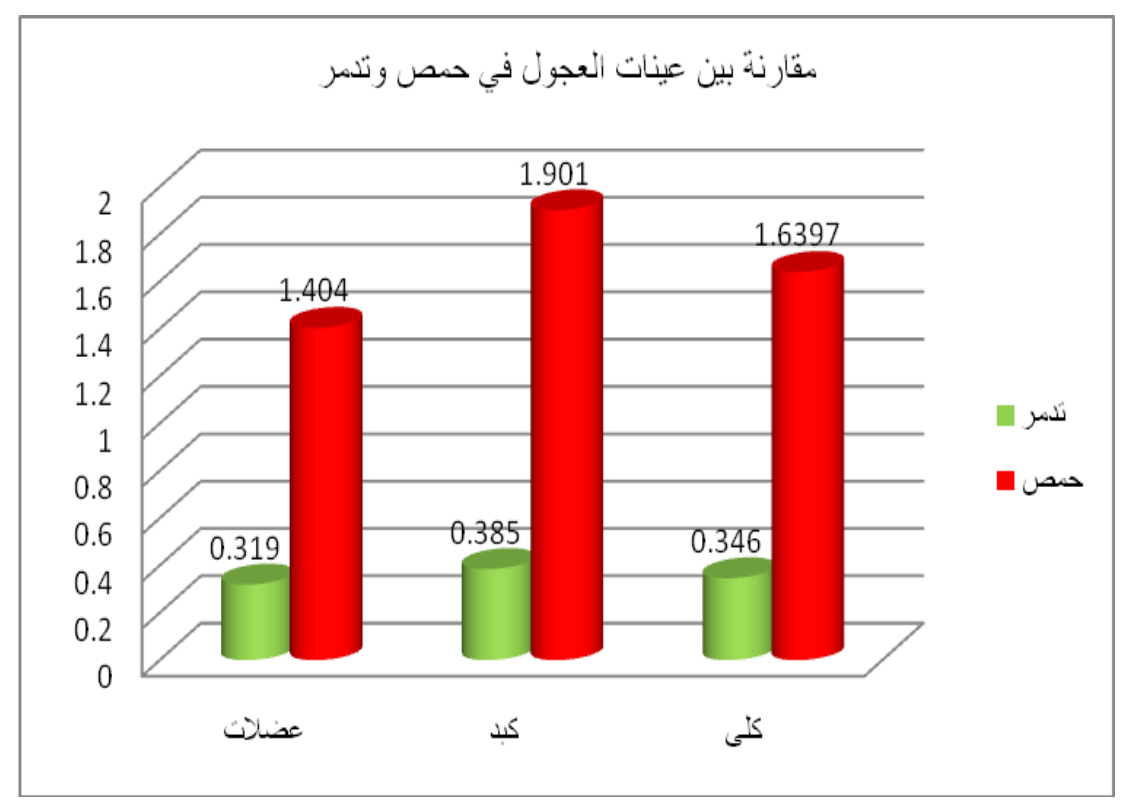

مخطط بياني رقم 2: مقارنة بين المتوسط الحسابي لعينات الكلع و الكبد و العضلات في

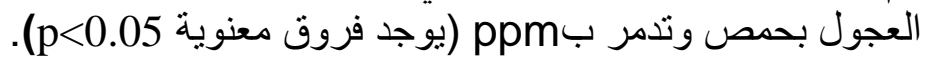


Assiut Vet. Med. J. Vol. 57 No. 128 January 2011

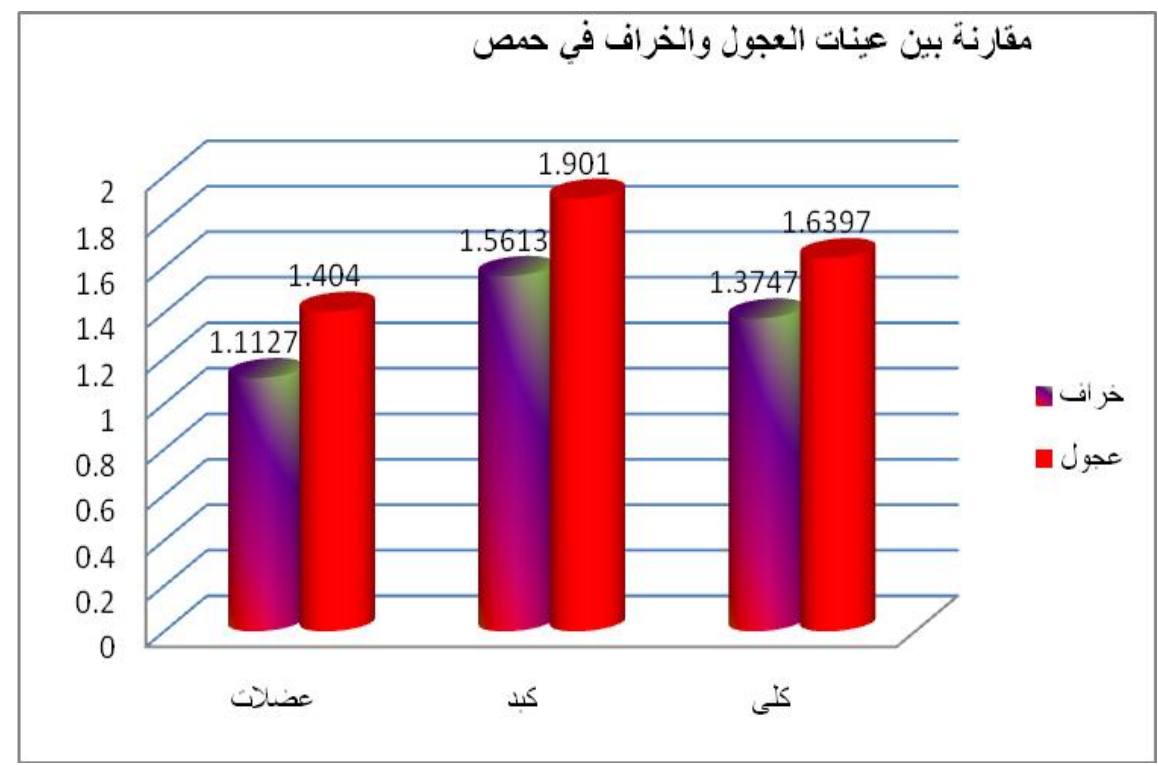

مخطط بياني رقم 3: مقارنة بين المتوسط الحسابي لعينات الكلى و الكبد و العضلا ت في

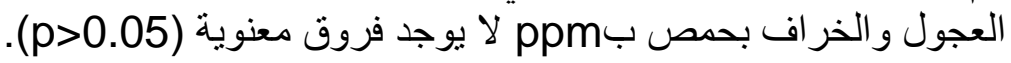

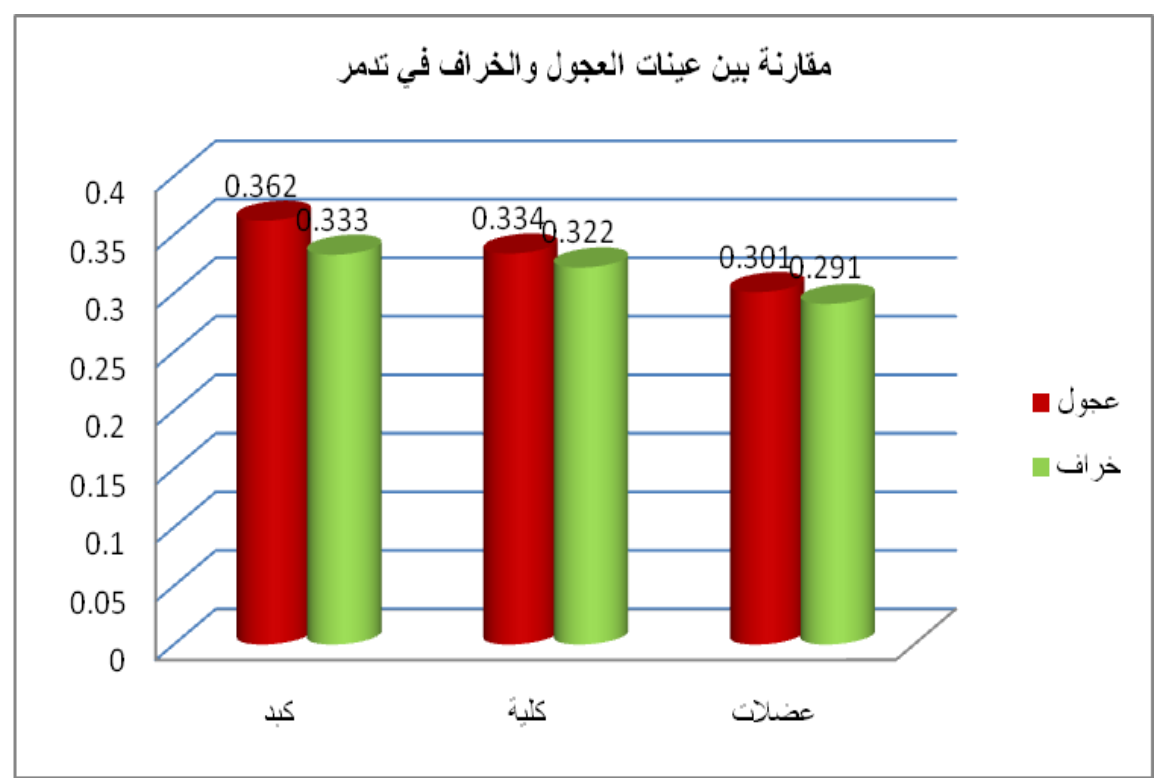

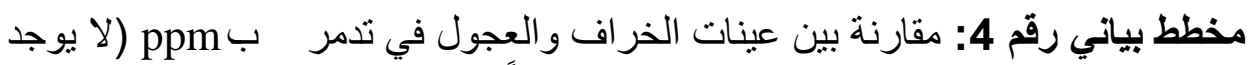

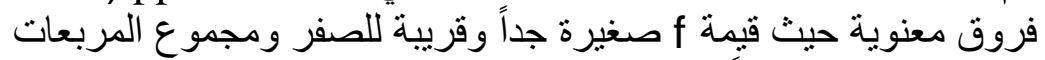

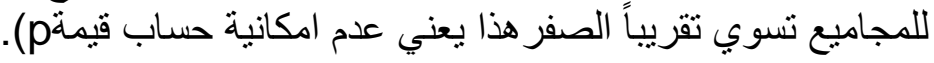


الجدول رقم 4: النسبة المئوية للعبنات الملوثة و السليمة في مختلف الأعضاء للعينات

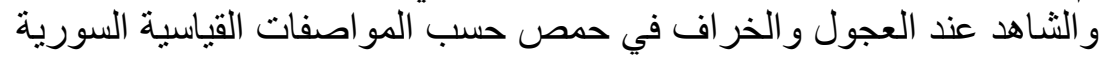

\begin{tabular}{|c|c|c|c|c|}
\hline الكبد & الكلية & العضلات & حال العينات & الحيوان \\
\hline$\% 80$ & $\% 70$ & $\% 90$ & ملوثة & \multirow[t]{2}{*}{ عجول حمص } \\
\hline$\% 20$ & $\% 30$ & $\% 10$ & سليمة & \\
\hline$\% 30$ & $\% 10$ & $\% 70$ & ملوثة & \multirow{2}{*}{ عجول تدمر } \\
\hline$\% 70$ & $\% 90$ & $\% 30$ & سليمة & \\
\hline$\% 83.33$ & \%76.66 & $\% 80$ & ملوثة & \multirow[t]{2}{*}{ خر اف حمص } \\
\hline$\% 16.67$ & $\% 23.34$ & $\% 20$ & سليمة & \\
\hline$\% 10$ & $\% 10$ & $\% 70$ & ملوثة & \multirow[t]{2}{*}{ خر اف تدمر } \\
\hline$\% 90$ & $\% 90$ & $\% 30$ & سليمة & \\
\hline
\end{tabular}

\section{DISCUSSION \\ المناقشــة}

أظهرت الدر اسة وجود اختلاف في تركيز بقايا عنصر الرصاص في في الأعضاء

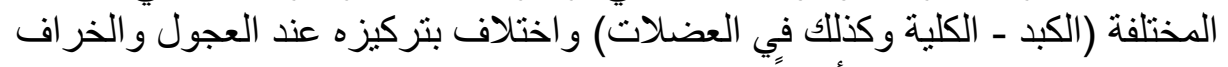
وفي عينات حمص وتدمر أيضاً. وقد سجلت القيم المرتفعة لتركيز الرصاص الرصاص في الأحشاء الداخلية (الكبد ، الكلية)

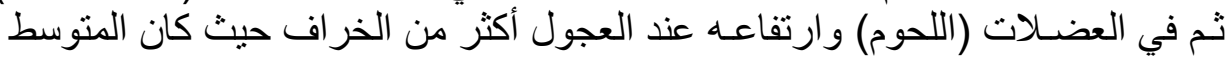

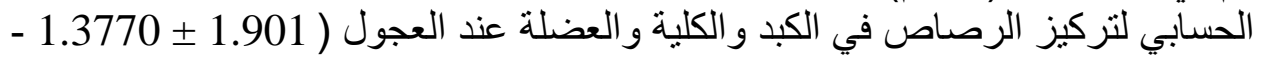

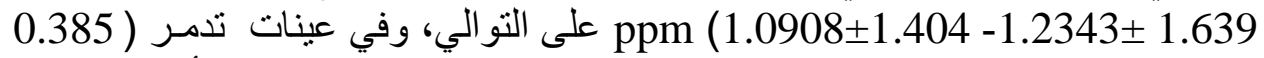

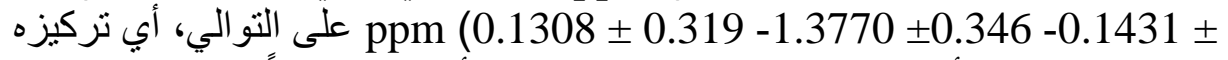

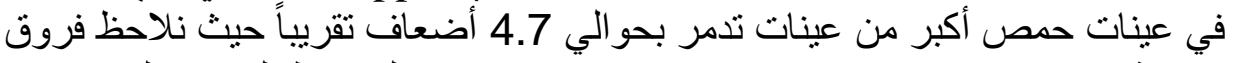

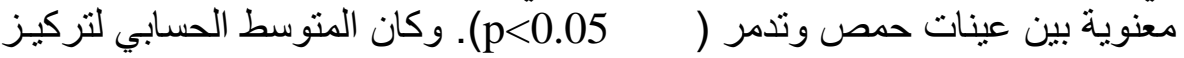

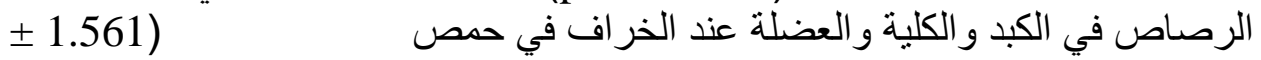

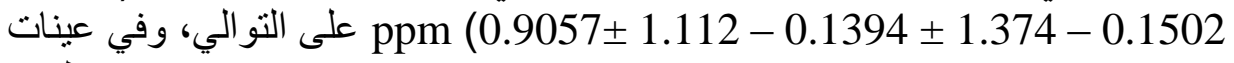

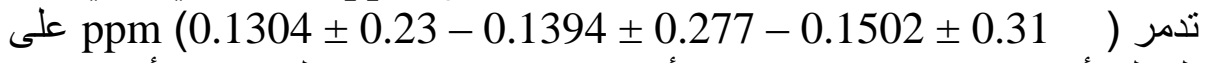
التو الي أي تركيزه في عينات حمص أكبر من عينات تدمر بحو التين

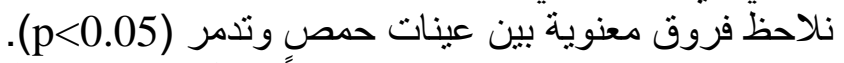

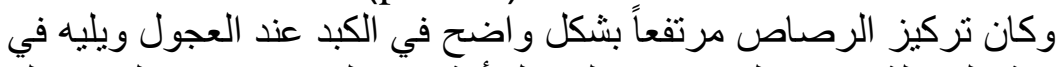

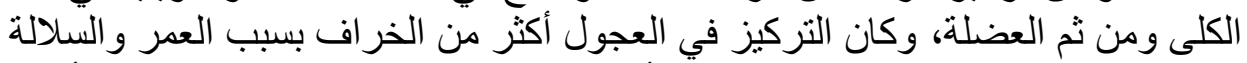

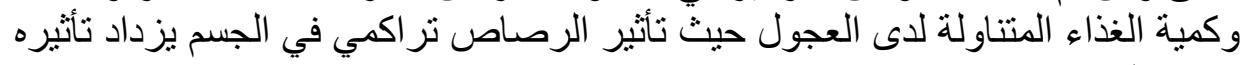

حسب العمر (Kosurd et al., 1985). 
ونسبة الرصاص كانت أعلى في الكبد و الكلية و العضلة في منطقة حمص أكثر

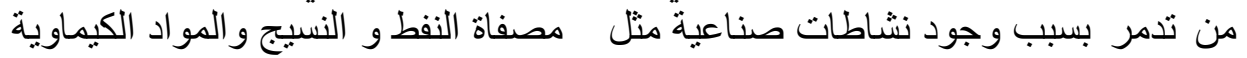

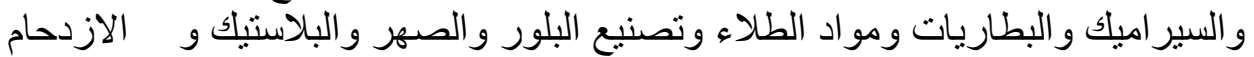

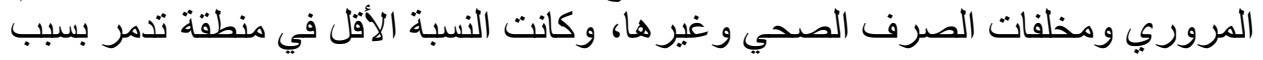

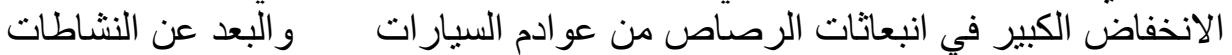

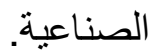

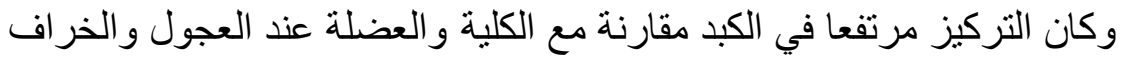

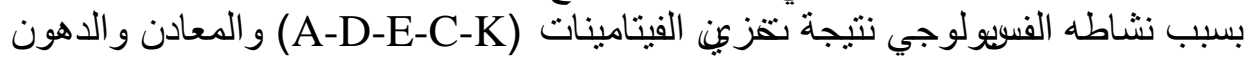

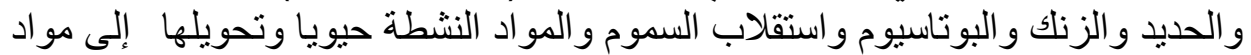

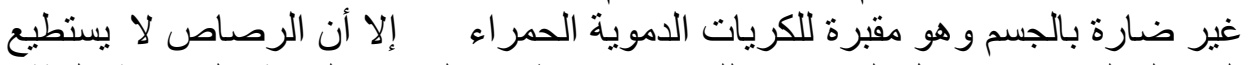

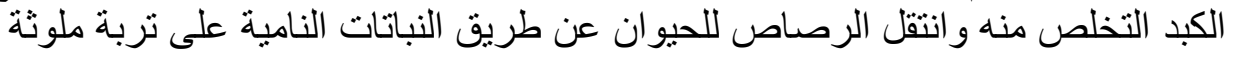
و إضافة المخصبات و المقويات الكيميائية والعضوية لئن عليها (Adebayo et al., 2009).

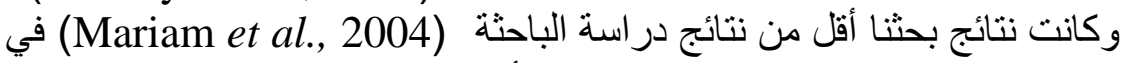

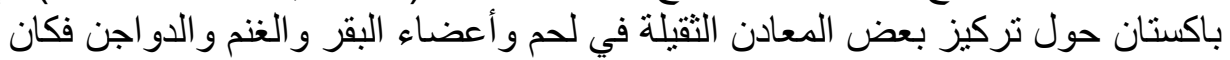
تركيز الرصاص في الكبد والكلية و اللحم عند البقر

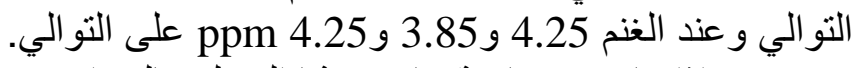

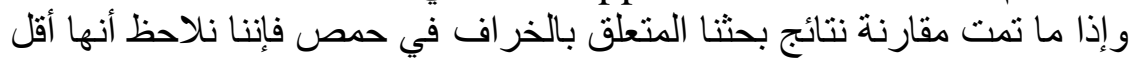

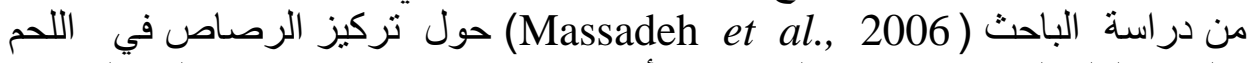

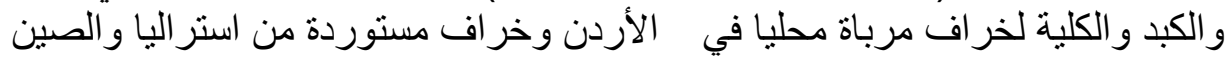

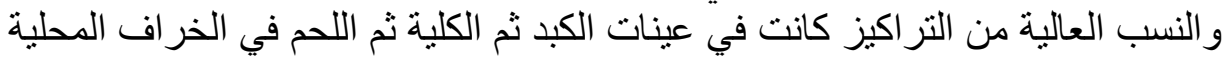

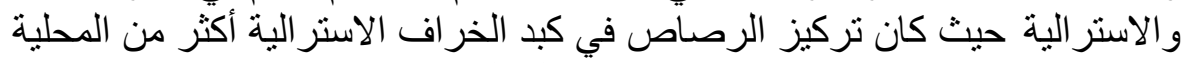

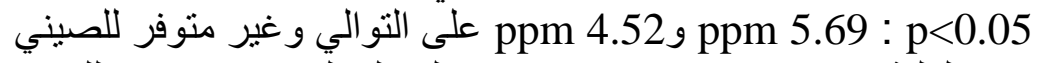

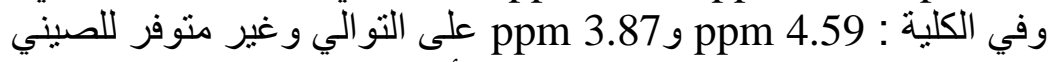

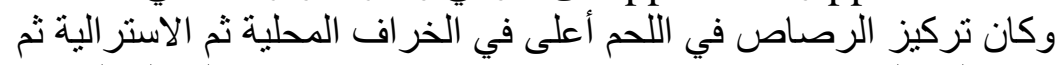

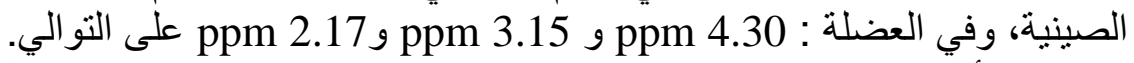
و أقل من نتائج الباحثة (Mariam et al., 2004) في باكستان حول الأن تركيز

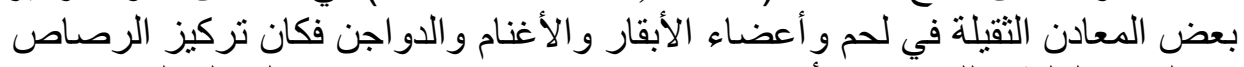

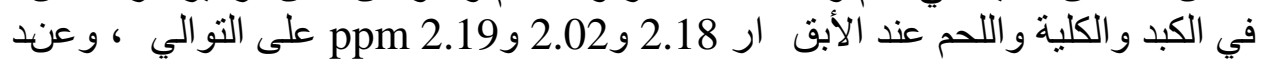

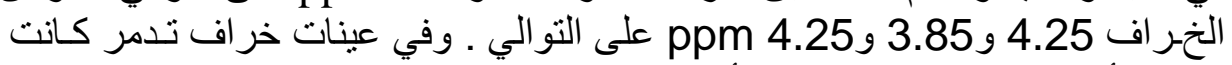

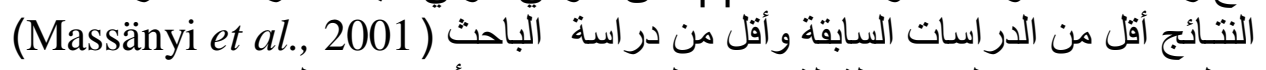

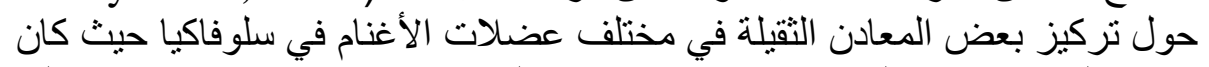

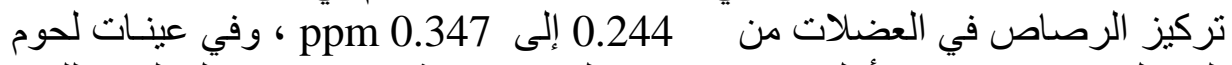

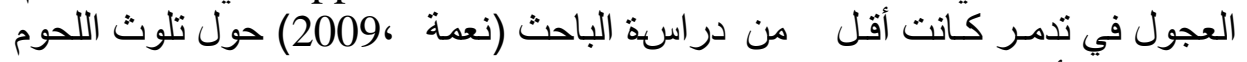

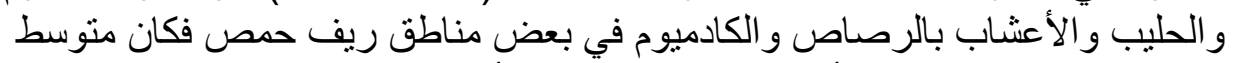

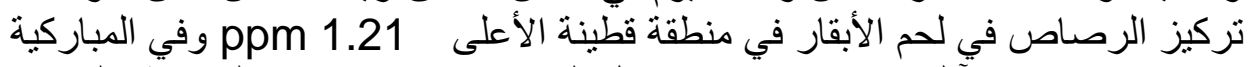

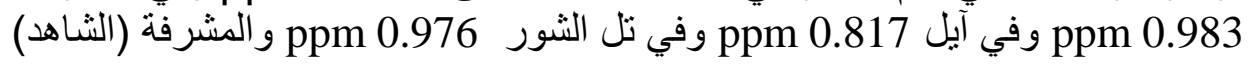
.ppm 0.482 
وتركيز الرصاص في عضلة العجول في تدمر كانت النتائج أقل من الدراسات

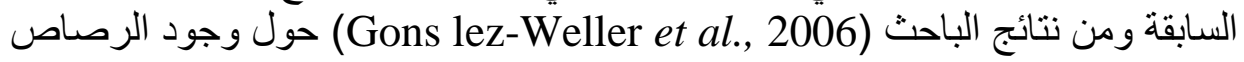

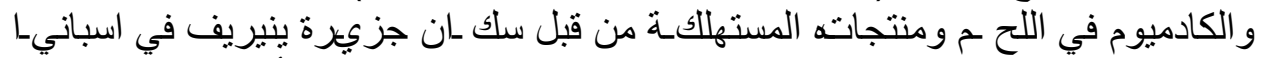

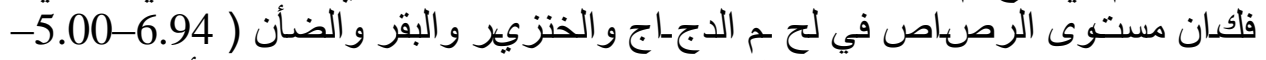

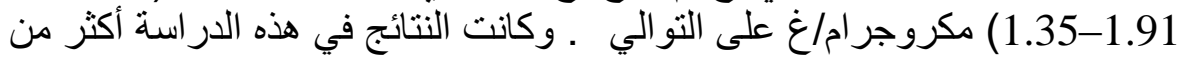

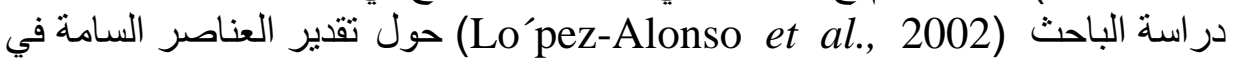

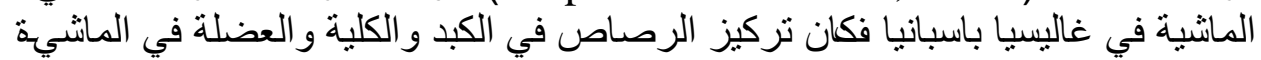

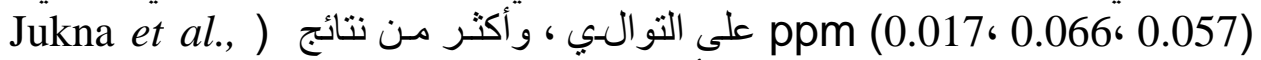

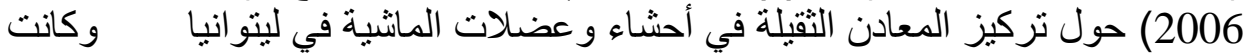

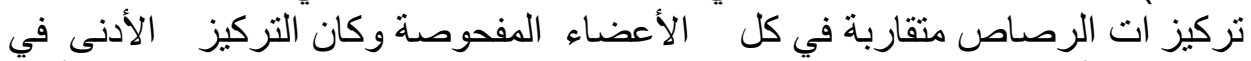

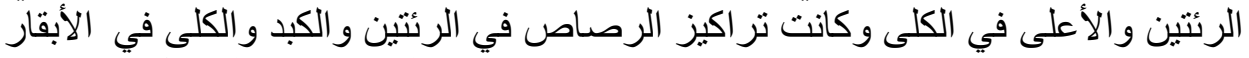
ع عالى التو الي وفي العجول المخصية

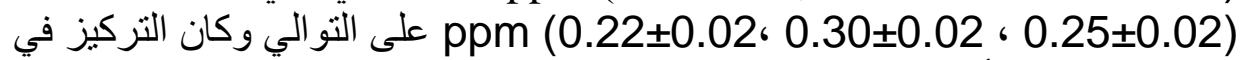

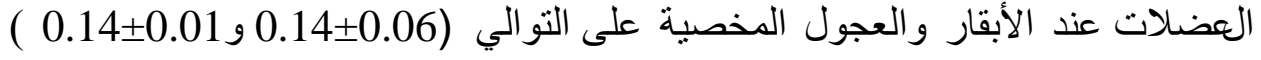

. ppm

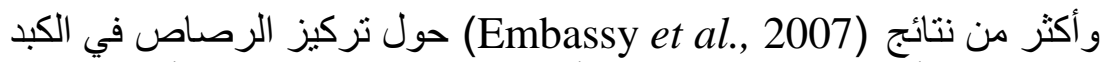

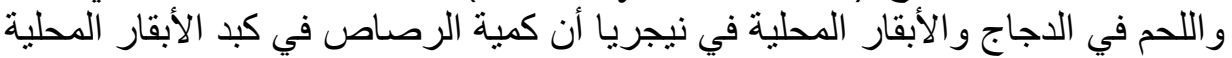

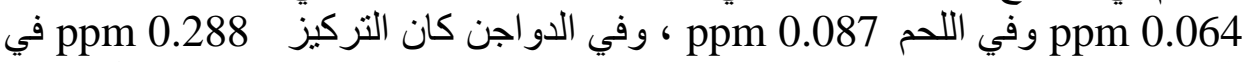

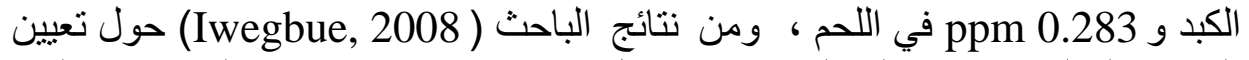

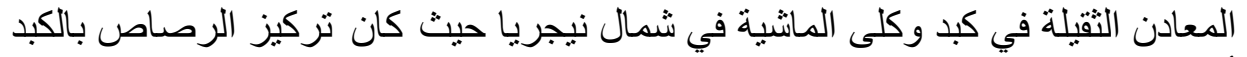

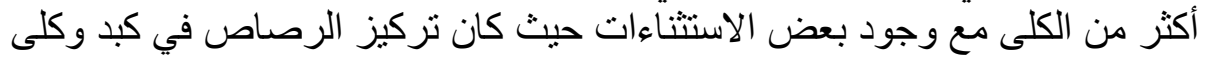

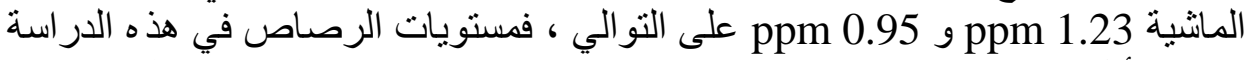

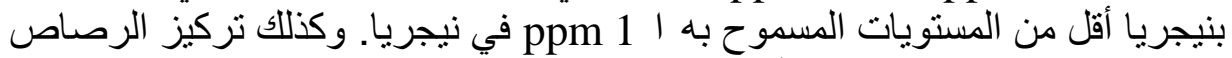

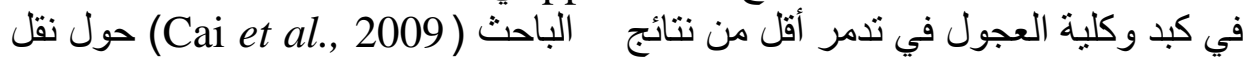

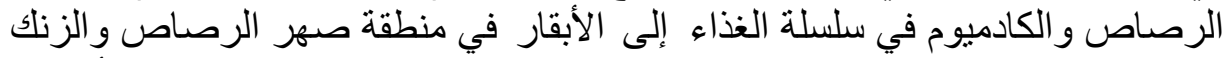

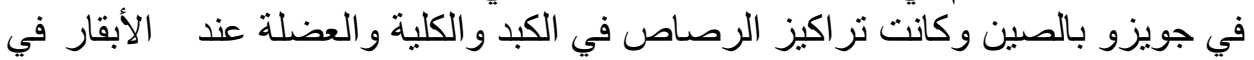

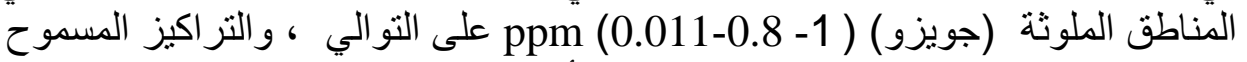

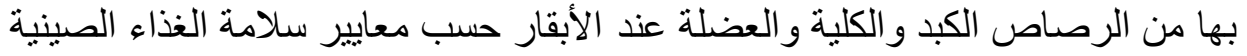
ع على التوالي. ppm (0.2-0.5-0.5)

\section{CONCLUSIONS الاستنتاج-ات}

نستنتج من هذا البحث أن نسب التلوث المئوية في نتائج در استنا في الكبد و الكلية

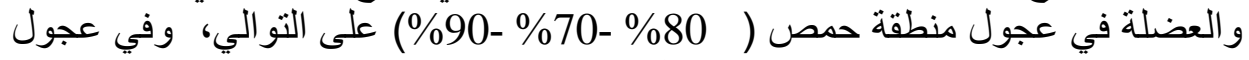

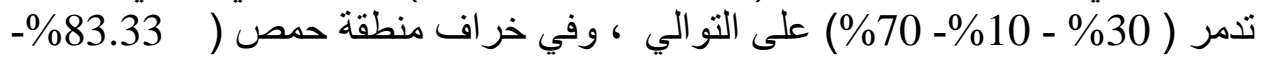




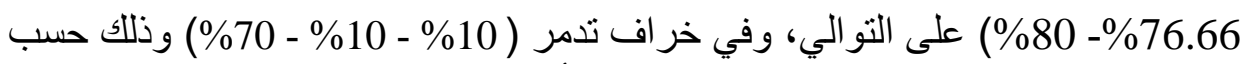

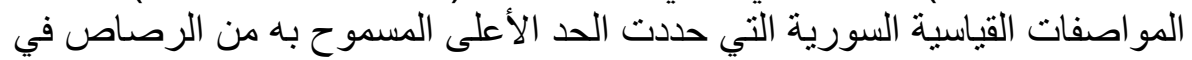

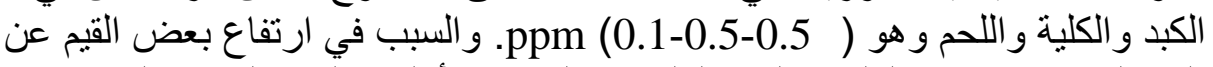

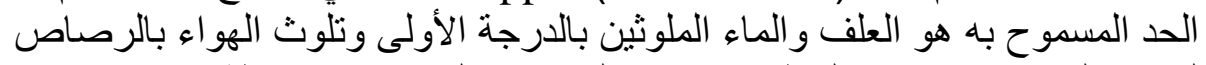

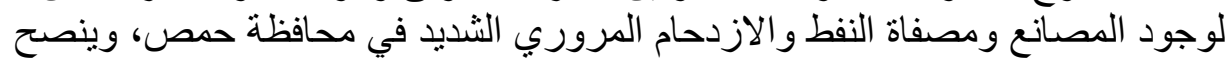

- بتغذية الحيو انات على نباتات ومواد علفية خالية من التلوث ومن أماكن بعيدة عن

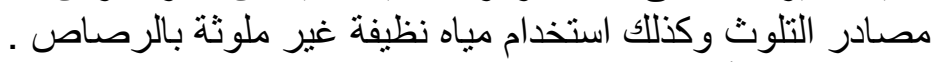

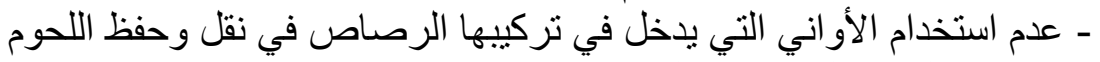

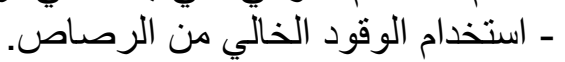

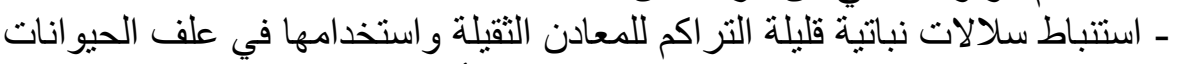

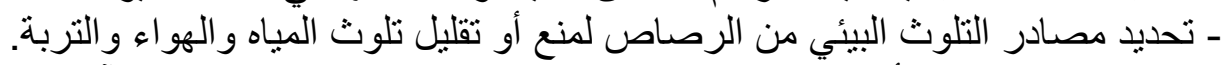

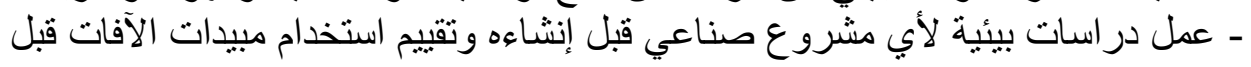
استخدامها.

\section{REFERENCES}

\section{المراجـع}

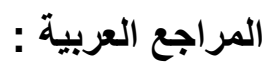

لجنة التفاوض الحكومبة الدولية (2004): الدورة الحادية عشرة، جنيف ،

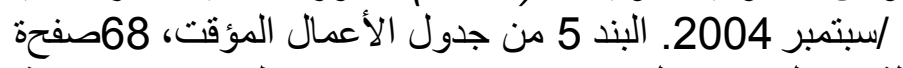

الأسبوع الثقافي الكبيبائي الخامس ( 5 من جدول 2003): دور الكيمياء في سلامة البيئة جامعة

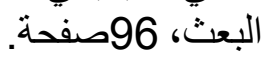

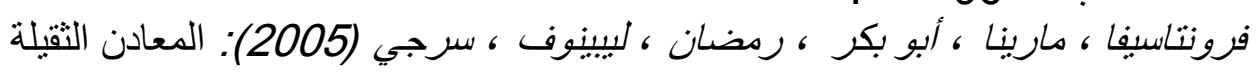

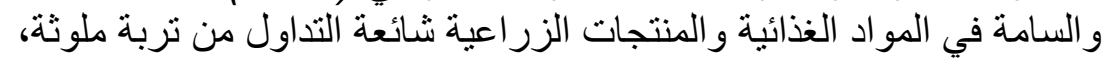

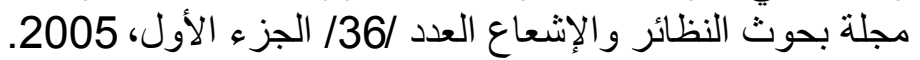

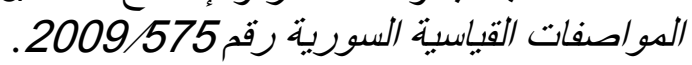

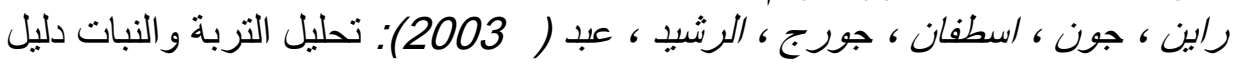

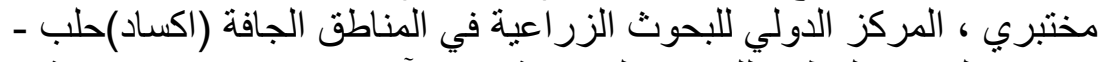

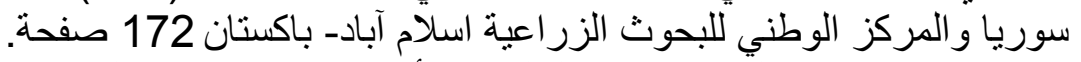

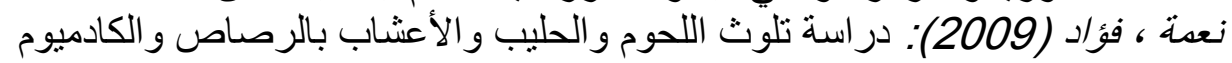

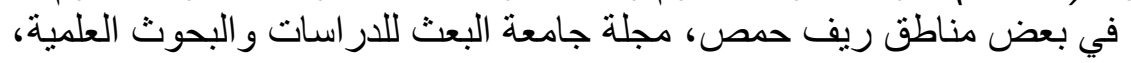

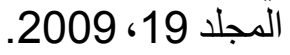

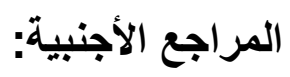

Adebayo, G.B.; Otunola, G.A. and Oladipo, F.O. (2009): Determination of Trace Elements in Selected Organs of Cow for Safety 
Consumption among Rural Dwellers in Kwara State, Nigeria, Pakistan Journal of Nutrition 8 (12): 1855-1857.

Cai, Q.; Li Long, L.; Zhu, M.; Zhou, Q.; Zhang, M. and Liu, J. (2009):

Food chain transfer of cadmium and lead to cattle in a lead-zinc smelter in Guizhou, China, Environmental Pollution 157: 3078-3082.

Eisinger, Josef-Sweet. (1996): Poison-Natural History Vol. 105 No. 7: 48-53.

El-Mowafi, A.F. (1995): Role of some mineral in fish nutrition. $\mathrm{PhD}$ thesis (Animal Nutrition), Faculty of Veterinary Medicine, Zagazig University, Egypt, 85-104.

Embassy, A.; Nok, A.J.; Oladipo, M.O.A.; Mohammed, A.; Saidu, S.A. and Bonire, J.J. (2007): Trace Metals Analysis in Tissues of Exotic and Locally Bred Cow, Chicken, and Pig in Zaria. Chem Class Journal, 4: 95-99.

Gonz Lez-Weller, D.; Karlsson, L.; Caballero, A.; Hernındez, F.; Gutirrez, A.; Gonz،lez-Iglesias, T.; Marino, M. and Hardisson, A. (2006): Lead and cadmium in meat and meat products consumed by the population in Tenerife Island, Spain, Food Addit Contam Aug.; 23(8): 757-63.

Iwegbue, C.M. (2008): Heavy metal composition of livers and kidneys of cattle from southern Nigeria Veterinarski Arhiv 78 (5): 401-410.

Jukna, C.; Jukna, V. and Siugzdaite, J. (2006): Determination of Heavy Metals In Viscera and Muscles of Cattle, Bulgarian Journal of Veterinary Medicine, 9, 1: 35-41.

Kosurd, G.O.; Meldrum, J.B.; Salibury, C.D.; Houlaham, B.J.; Saschenbreaker, P.W. and Tittiger, F. (1985): Can. J. Comp. Med. 49(2): 159-163.

Lo'pez-Alonso, M.; Benedito, JL.; Miranda, M.; Castillo, C.; Herna'ndez, $J$. and Shore, RF. (2002): Cattle as biomonitors of soil arsenic, copper and zinc concentrations in Galicia (NW Spain). Arch. Environ. Contam Toxicol, 43: 103-8.

Maldonado, V.M.; Cerbon, S.J.; Albores, M.A; Hernandez, L.C. and Calderonsalinas, J.V. (1996): Lead: intestinal absorption and bone mobilization during lactation. Hum. Exp. Toxicol., 15: $872-7$.

Mariam, I.; Iqbal, SH. and Nagra, S.A. (2004): Distribution of Some Trace and Macrominerals in Beef, Mutton and Poultry. International Journal of Agriculture and Biology 15608530/2004/06-5-816-820. 
Massadeh, A.; AL-Sharif, L. and Dalale'h, R. (2006): Analysis of Lead Levels In Local Jordanian Andimported Sheep Meat And Organs Using Atomic absorption Spectrometry, (JUST), Irbid, Jordan Environmental Monitoring and Assessment 115: 87-93.

Massänyi, P.; Nad, P.; and Koväcik, J. (2001): Concentrations of Cadmium, Lead, Nickel, Copper and Zinc in Various Museies of Sheep, Die Bodenkultur 52 (3) 255-258.

Miranda, M.; Lo'pez-Alonso, M.; Castillo, M.; Herna'ndez, J. and Benedito, J.L. (2005): Effects of moderate pollution on toxic and trace metal levels in calves from a polluted area of northern Spain, Environment International 31: 543-548.

Official Methods of Analysis of the Association of official Analytical Chemists, A.O.A.C. (1990): Washington, DC.

Seady, N.I. (2001): Evaluation of Heavy Metals in Meat and Offal of Various Animal Speciesslaughtered in Menoufia Governorate. Ph.D. Thesis, Faculty. Vet. Med., Moshtohor, Zagazig University (Benha Branch).

Tsoumbaris, P. (1990): Heavy Metals Determination in Foodstuff, PhD Thesis, Thessaloniki, Greece.

Geneva, W.H.O. (2000): IPCS - International Program on Chemical safety contaminations.

Wright, R.O.; Tsaih, S.W.; and Schwartz, J.( 2003 ): Associationbetween iron deficiency and blood lead level in longitudinal analysis of children followed in an urban primary care clinic. J. Pediatric 142: p. $9-14$.

Zantopoulos, N.; Antoniou, V.; Petsaga, V. and Zdragas, A.: (1996): 'Copper concentrations in sheep liver and kidney in Greece', Vet. Hum. Toxicol, 38(3): 184-185. 